\title{
The p63 target HBP1 is required for skin differentiation and stratification
}

\author{
S Borrelli ${ }^{1}$, E Candi ${ }^{2}$, B Hu ${ }^{3}$, D Dolfini ${ }^{1}$, M Ravo ${ }^{4}$, OMV Grober $^{4}$, A Weisz ${ }^{4,5}$, GP Dotto ${ }^{3}$, G Melino ${ }^{2,6}$, MA Viganò ${ }^{1}$ and R Mantovani, ${ }^{*}, 1$
}

Genetic experiments established that p63 is crucial for the development and maintenance of pluristratified epithelia. In the RNA interference (RNAi) screening for targets of p63 in keratinocytes, we identified the transcription factor, High Mobility Group (HMG) box protein 1 (HBP1). HBP1 is an HMG-containing repressor transiently induced during differentiation of several cell lineages. We investigated the relationship between the two factors: using RNAi, overexpression, chromatin immunoprecipitations and transient transfections with reporter constructs, we established that HBP1 is directly repressed by p63. This was further confirmed in vivo by evaluating expression in p63 knockout mice and in transgenics expressing p63 in basal keratinocytes. Consistent with these findings, expression of HBP1 increases upon differentiation of primary keratinocytes and $\mathrm{HaCaT}$ cells in culture, and it is higher in the upper layers of human skin. Inactivation of HBP1 by RNAi prevents differentiation of keratinocytes and stratification of organotypic skin cultures. Finally, we analyzed the keratinocyte transcriptomes after HBP1 RNAi; in addition to repression of growth-promoting genes, unexpected activation of differentiation genes was uncovered, coexisting with repression of other genes involved in epithelial cornification. Our data indicate that suppression of HBP1 is part of the growth-promoting strategy of p63 in the lower layers of epidermis and that HBP1 temporally coordinates expression of genes involved in stratification, leading to the formation of the skin barrier.

Cell Death and Differentiation (2010) 17, 1896-1907; doi:10.1038/cdd.2010.59; published online 4 June 2010

p63 is a transcription factor (TF) homologous to p53 and p73. p53 directs the response to DNA damage by impinging on cell-cycle control and pro-apoptotic pathways, ${ }^{1}$ whereas p63 is involved in the development and maintenance of pluri-stratified epithelia. ${ }^{2}$ Six p63 proteins have been described, resulting from two distinct promoters - amino-terminal transcriptional activation domain-containing p63 (TAp63) and transcriptional activation domain-deleted p63 $(\Delta \mathrm{Np} 63)-$ and from alternative mRNA splicing $-\alpha, \beta$ and $\gamma-$ at the $3^{\prime}$ end of the gene. TAp63 contains a transcriptional activation domain that is missing in the $\mathrm{N}$-terminally deleted $\Delta \mathrm{Np} 63$ isoforms; differential splicing generates isoforms with or without a sterile $\alpha$-motif (SAM) domain, allegedly implicated in protein-protein interactions. The relative properties and functions of these isoforms are still not fully understood, but the major isoform present in keratinocytes and other multi-layered epithelia $-\Delta \mathrm{Np} 63 \alpha$ - is essential for ectodermal development in zebrafish, as well as in mammals. Indeed, the importance of p63 in skin development is shown by mice lacking p63, which die soon after birth with severe defects in limb and craniofacial development and absence of skin. In humans, several syndromes showing abnormalities in limbs, skin and epithelial annexes are caused by missense mutations in the 063 gene. ${ }^{3}$ p63 is crucial for the activation of the epithelial cell adhesion program, and it has a major role in maintaining the proliferative potential of stem cells of the multilayered epithelia.

During a profiling screening for p63 targets by RNA interference (RNAi)-mediated functional inhibition, we identified the High Mobility Group (HMG) box protein 1 (HBP1) as a potential target. First identified in rat brain, on the basis of its ability to suppress the potassium transport-defective phenotype of mutant Saccharomyces cerevisiae, HBP1 was later rediscovered in yeast two hybrids screenings for $\mathrm{Rb}$-interacting proteins and in one hybrid assays for TFs that bind to histone $\mathrm{H} 1$ promoters. It is a member of the HMG protein family, containing a 70 amino-acid HMG box DNA-binding domain that mediates sequence-specific binding. HBP1 was mainly characterized as a transcriptional repressor, a function provided by the central Ataxin - $\mathrm{AXH}$ - domain, and an important regulator of cell-cycle exit, apoptosis and terminal differentiation. ${ }^{4}$ However, transcriptional activation by HBP1 was also reported, notably on the tissue-specific $\mathrm{H} 1$, MPO and CD2 promoters. Overexpression induces significant changes in the mRNA levels of important TFs involved in differentiation

\footnotetext{
${ }^{1}$ Dipartimento di Scienze Biomolecolari e Biotecnologie, Università degli Studi di Milano, Via Celoria 26, 20133 Milano, Italy; ${ }^{2}$ Biochemistry IDI-IRCCS laboratory, c/o University of Rome 'Tor Vergata', Via Montpellier 1, 00133 Roma, Italy; ${ }^{3}$ Department of Biochemistry, University of Lausanne, Chemin des Boveresses 155, Epalinges, CH-1066, Switzerland; ${ }^{4}$ Dipartimento di Patologia Generale and Centro Grandi Apparecchiature, Seconda Università di, Napoli, vico L. De Crecchio 7, 80138 Napoli, Italy; ${ }^{5}$ AIRC Naples Oncogenomics Centre, c/0 CEINGE Biotecnologie Avanzate, via Comunale, Margherita 482, 80145 Napoli, Italy and ${ }^{6}$ Medical Research Council, Toxicology Unit, Hodgkin Building, Leicester University, Lancaster, Road, P.O. Box 138, Leicester LE1 9HN, UK

*Corresponding author: R Mantovani, Dipartimento di Scienze Biomolecolari e Biotecnologie, Università degli Studi di Milano, Via Celoria 26, Milano 20133, Italy. Tel: + 39025031 5005; Fax: + 39025031 5044; E-mail: mantor@ unimi.it Keywords: HBP1; p63; keratinocytes

Abbreviations: TAp63, amino-terminal transcriptional activation domain-containing p63; $\Delta \mathrm{Np} 63$, transcriptional activation domain-deleted p63; ChIP, chromatin immunoprecipitation; HBP1, High Mobility Group box protein 1; RNAi, RNA interference; NHEK, normal human epidermal keratinocyte; KRT, keratin; tg, transgenic mice; KO, knockout mice; TF, transcription factor; SAM, sterile $\alpha$-motif; SC, stratum corneum; EMT, epithelial mesenchimal transition

Received 13.7.09; revised 24.2.10; accepted 25.2.10; Edited by RA Knight; published online 04.6.10
} 
of myeloid cells: C/EBP $\alpha$, JunB, GATA-1 and RUNX1 are increased, and c-Myb, c-Myc and PU.1 are decreased. ${ }^{5}$ These changes are in agreement with the idea that HBP1 is a growth-suppressive, pro-differentiation TF. In line with this, the gene is induced during differentiation in a variety of tissues and cell types, and the protein inhibits cell-cycle progression. The molecular mechanisms were also investigated, and direct inhibition of transcriptional activation of Wnt target genes, such as cyclin D1 and c-MYC, was detected. Two additional sets of data link HBP1 to growth suppression of tumor cells. First, HBP1 was shown to be required for oncogene-mediated premature senescence, which is a feature that is lost upon malignant transformation. ${ }^{6}$ Second, Yee et al. ${ }^{7}$ recently detected two types of HBP1 abnormalities in a large set of breast cancers: the presence of ill-defined variants/mutants and complete lack of expression in a statistically significant number of tumors, indicating a role of HBP1 suppression in the genesis of this type of neoplasia.

Essentially nothing is known about the role of HBP1 in keratinocytes and its link to p63. Our interest was enhanced by the finding that p63 has the propensity to target many, if not all TFs genes involved in growth/differentiation decisions. We decided to characterize the relationship between the two TFs by using a variety of in vitro and in vivo experiments.

\section{Results}

p63 represses HBP1. Affymetrix microarray profiling of HaCaT cells, in which 063 was silenced by siRNA, ${ }^{10}$ identified HBP1 as a gene repressed by p63 (Affymetrix, Santa Clara, CA, USA). We validated it as a target by RT-PCR in $\mathrm{HaCaT}$ and primary normal human epidermal keratinocytes (NHEKs) after p63 RNAi; the decrease of p63 led to an increase of HBP1 mRNA, compared with cells transfected with the scrambled RNAi, and the housekeeping GAPDH was indeed invariant (Figure 1a). We overexpressed the predominant isoform present in keratinocytes $-\Delta \mathrm{Np} 63 \alpha-$ as well as TAp63 $\alpha$ and analyzed endogenous HBP1 mRNA levels using semiquantitative RT-PCR (Figure 1b). To appreciate the overexpression of the transfected genes, we had to limit the cycles of PCR amplifications, which prevented the endogenous levels of p63 to show up; along with the robust overexpression of p63, HBP1 is indeed reduced, particularly with $\Delta \mathrm{Np} 63 \alpha$. We conclude that the HBP1 gene is repressed by p63. To assess whether this is a direct effect, we performed chromatin immunoprecipitation (ChIP) assays using anti-p63 and control antibodies on the HBP1 promoter region. We selected an amplicon in the core promoter in which conservation between human and mouse was high and a consensus p53/p63 was found using bioinformatic means; the anti-p63 antibody was positive in HaCaT and NHEK chromatin (Figure 1c). Finally, we tested the transcriptional effect of the six p63 isoforms on the activity of the HBP1 promoter $(-1.2$ to $+50 \mathrm{~Kb})$ fused to a luciferase reporter, by transiently transfecting NHEK cells. As shown in Figure 1d, all p63 isoforms efficiently repress the promoter. Altogether, these data indicate that HBP1 is under direct negative regulation of p63 in human keratinocytes.

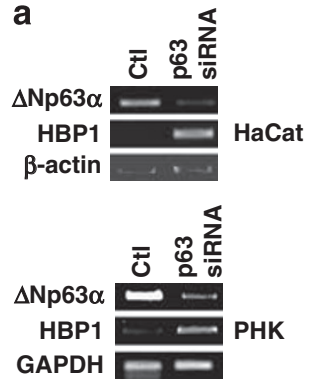

b
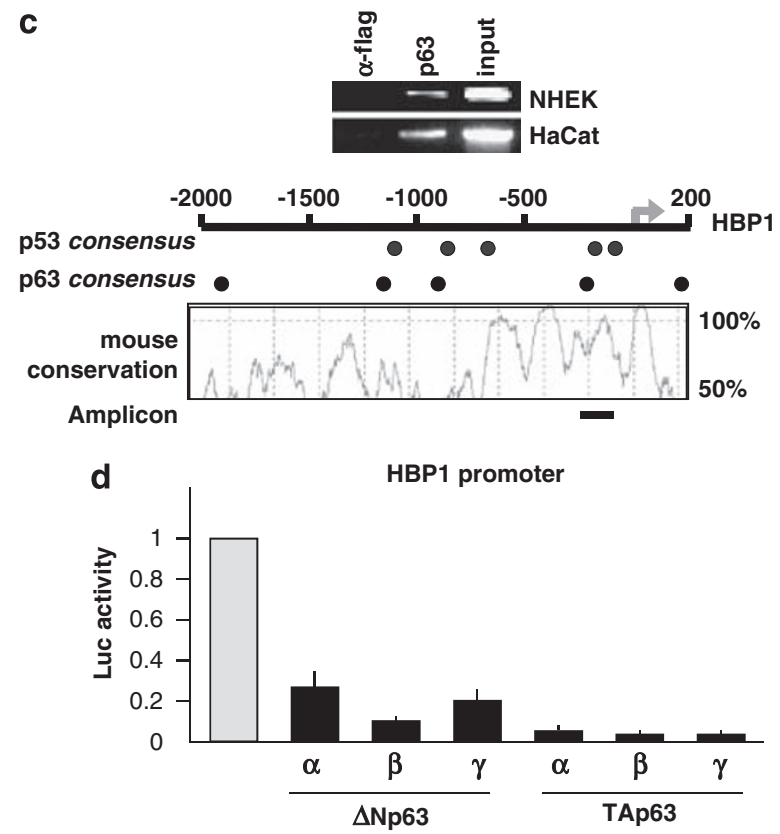

Figure $1 \mathrm{HBP} 1$ is a target of $\mathrm{p} 63$. (a) Expression analysis of the indicated genes in HaCaT and NHEK cells, treated with siRNA p63 or scramble oligo (Ctl). (Upper panels) RT-PCR with RNA extracted from $\mathrm{HaCaT} ; \beta$-actin was used to normalize cDNA. (Lower panel) RT-PCR with RNA extracted from NHEK; GAPDH was used to normalize. (b) p63 overexpression in NHEK. RT-PCR analysis of the indicated genes with RNA extracted from NHEK $48 \mathrm{~h}$ after transient transfection with $\triangle N p 63 \alpha$ and TAp63 $\alpha$ expression plasmids. The normalization of the CDNAs was performed with GAPDH. (c) Chromatin immunoprecipitation analysis of $\mathrm{HaCaT}$ (upper panel) and NHEK (lower panel) with $\alpha$-p63 and control $\alpha$-Flag antibodies on the HBP1 promoter. The lower chart is a schematic representation of the HBP1 promoter locus, with black and gray dots indicating in silico p63 and p53 sites, respectively, in the appropriate position. The mouse conservation from CONSITE is also shown. The black bar indicates the region amplified in the ChIP experiments. (d) Transactivation assay in NHEK cells. Transcription activity of the different p63 isoforms co-transfected with the human HBP1 promoter. Bars represent the luciferase activity ( \pm S.D.), expressed as repression of the promoter activity co-transfected with an empty vector (Ctl)

Expression of HBP1 in wild type (wt), p63 knockout (KO) mice and in $\Delta \mathrm{Np63} \alpha$ and TAp63 $\alpha$ transgenics. To further explore the relationship between p63 and HBP1, we analyzed the expression of HBP1 in the skin of 19.5-dayold wt and p63 KO mouse embryos. In wt p63 mice, HBP1 is expressed in the nuclei of the granular and, to some extent, in the corneum layers, in which p63 is not expressed (Figure 2a, left panels). Some overlap with p63 is evident in the spinous layer. It is also expressed in the derma, which is expected, as the gene is known for having little tissue 
a
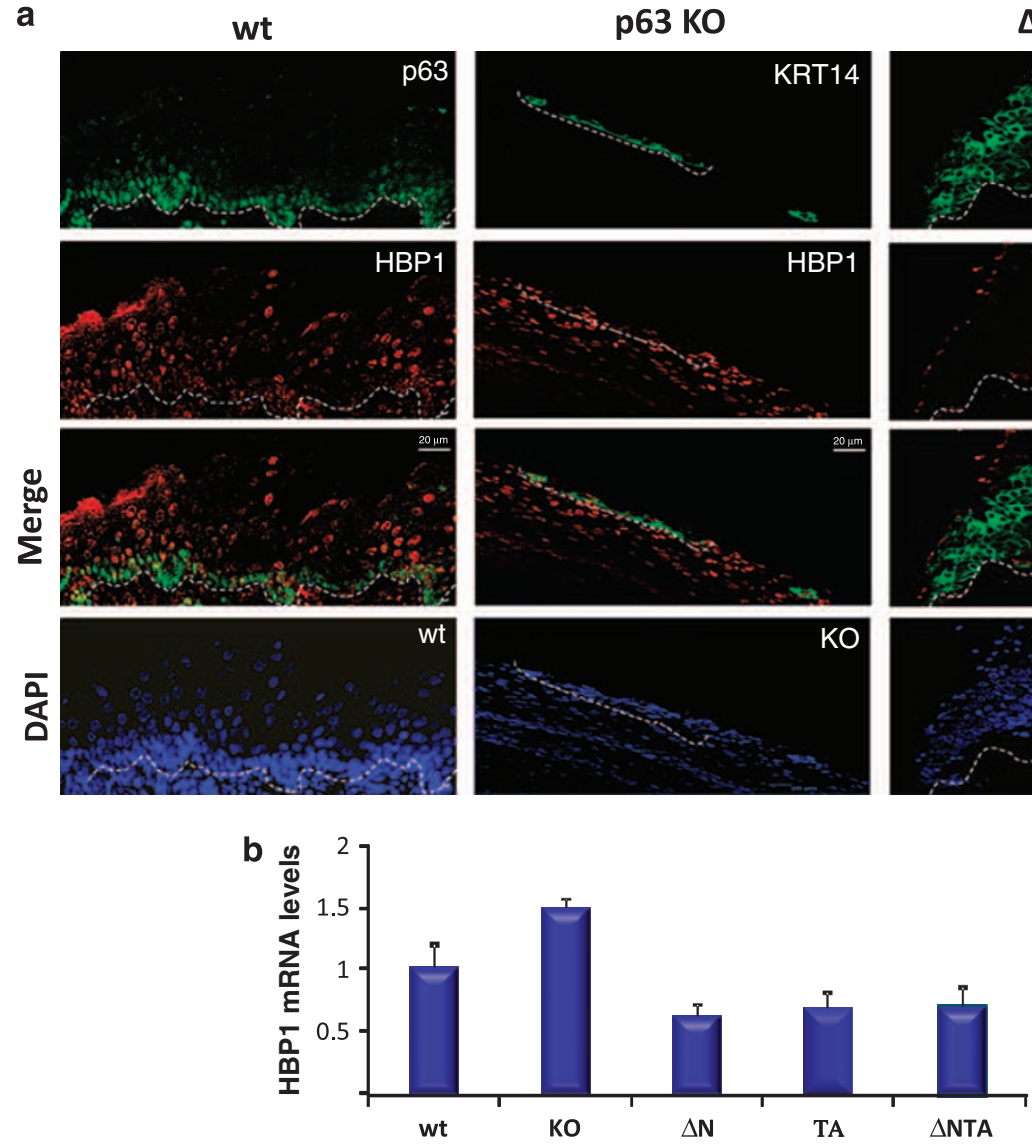

Figure 2 Expression of HBP1 in wt, p63 KO mice, $\triangle \mathrm{Np63} \alpha$ and TAp63 $\alpha$ transgenics. (a, left panels) Confocal images of mouse embryo skin immunostained with p63 (green) and HBP1 (red). (Middle panels) p63 ${ }^{-1-}$ mouse skin immunostained with KRT14 (green) and HBP1 (red). (Right panels) $\Delta$ Np63-Tg mice immunostained with KRT14 (green) and HBP1 (red). The bottom panels in each condition represent DAPI stainings. (b) qRT-PCR of mRNA extracted from the embryo skin of wt, p63 KO mice and from transgenics reconstituted with $\triangle \mathrm{Np} 63 \alpha, \mathrm{TAp} 63 \alpha$ and $\Delta \mathrm{N}-\mathrm{TAp} 63 \alpha$ genes under the control of the KRT5 promoter (see Zhang et al. ${ }^{25}$ )

preference. p63-null mice have scattered skin patches, identified by staining with anti-KRT14, a marker of the basal cells (Figure 2a, middle panels); in addition to the staining in the derma, unaffected by the p63 configuration, we detected high expression of HBP1, proportional to the levels of KRT14. Importantly, HBP1 is nuclear in p63 $\mathrm{KO}$ epithelial cells (Figure 2a, middle panels). Finally, we analyzed transgenic mice expressing $\Delta$ Np63 $\alpha$ under the control of the keratin 5 (KRT5) promoter, in a background of p63-null mice; these mice show some degree of re-epithelialization and re-express several differentiation markers. ${ }^{9}$ As shown in the right panels of Figure 2a, HBP1 expression is extremely low in the KRT14 ${ }^{+}$cells, which are also $\mathrm{p} 3^{+}$(see Supplementary Figure S1); moreover, in the few positive cells, it is localized in the cytoplasm. The lower panels show DAPI staining. To support the immunofluorescence studies, we performed qRT-PCR of the wt, $\mathrm{KO}$ and mouse skin embryos reconstituted with the $\Delta \mathrm{Np} 63$ and TA-p63 isoforms, and of the double transgenics. Figure $2 \mathrm{~b}$ shows an increase in HBP1 expression with p63 KO mRNA, compared with wt p63 mice, and a decrease in all the transgenic lines re-expressing p63. Taken together, these data indicate that $\mathrm{p} 63, \Delta \mathrm{N}$ or $\mathrm{TA}$, is a major negative regulator of HBP1 expression in keratinocytes in vivo.
HBP1 is activated upon keratinocyte differentiation. HBP1 has been shown to be regulated in several differentiation systems; we wished to ascertain whether this is the case in keratinocytes. NHEKs are differentiated in vitro by appropriate conditions that include addition of calcium to the medium: an analysis using semiquantitative RT-PCR indeed shows a substantial increase of HBP1 mRNA after differentiation (Figure 3a); a marker of upper layers such as KRT1 is upregulated in differentiated cells, whereas the basal KRT14 decreases substantially. The invariant NF-YB mRNA was used as an internal control. Next, we monitored using qRT-PCR the kinetics of HBP1 and p63 mRNA during the process. Figure $3 b$ shows a clear increase in HBP1, whereas p63 decreases, as expected. As a control, we also performed western blot analysis of keratinocyte differentiation (Supplementary Figure S2, panel A) Multiple bands in addition to the expected $50 \mathrm{kD}$ band are observed, collectively increasing upon differentiation; the decrease in p63 levels is evident, and loricrin expression levels are induced at the late stages of differentiation. We verified whether p63 binding to the HBP1 promoter is altered during differentiation, by performing ChIPs before and after 7 days of differentiation. Supplementary Figure S2, panel B shows that p63 is no longer present on the HBP1 promoter after 
a

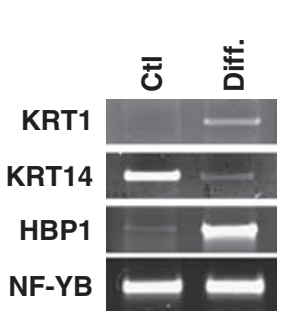

b

b

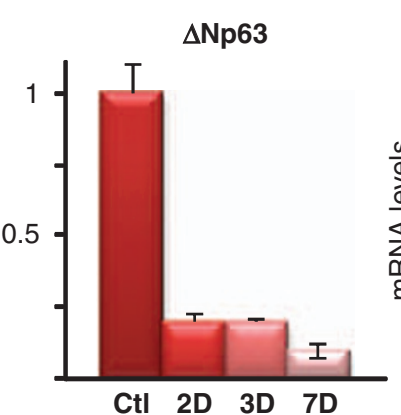

Ctl 2D 3D 7D

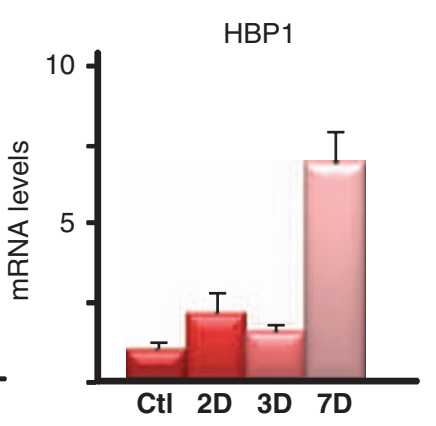

DAPI p63
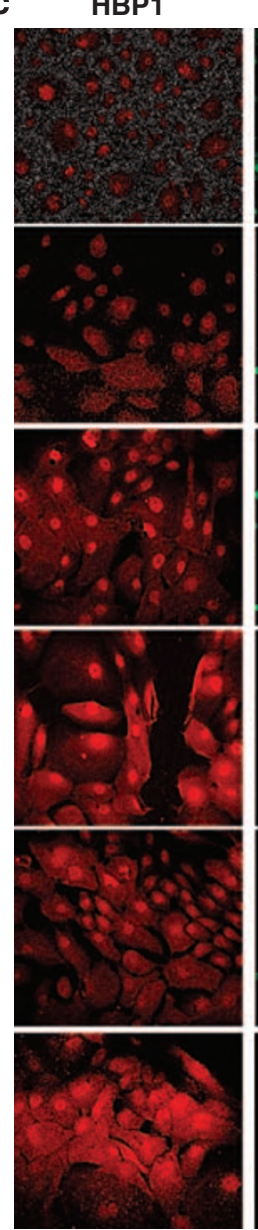
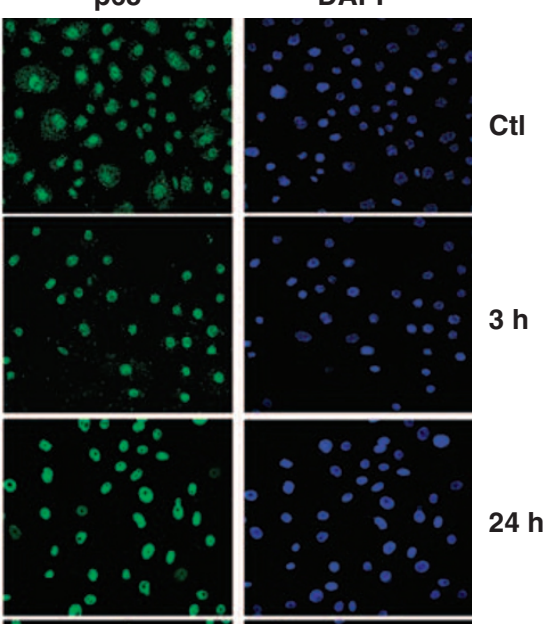

$3 \mathrm{~h}$
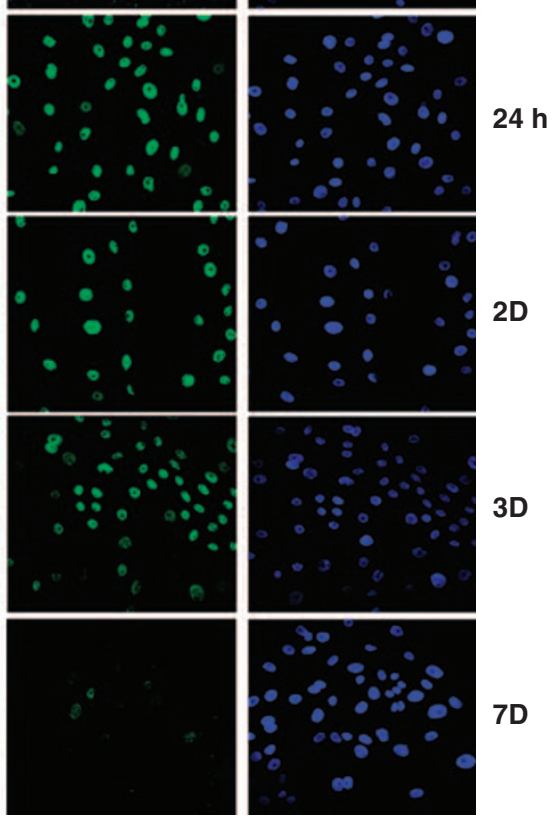

2D

3D

7D

Figure 3 HBP1 is activated upon keratinocyte differentiation. (a) Semiquantitative analysis of KRT1, KRT14, HBP1 and of the invariant control NF-YB in growing and differentiated NHEKs. (b) Kinetic analysis of $\triangle \mathrm{Np63}$ and HBP1 expression by qRT-PCR after 2, 3 and 7 days (2D, 3D and 7D) of differentiation. (c) Confocal immunofluorescence analysis of p63 and HBP1 expression in NHEKs after the indicated time following the differentiation stimulus

7 days, in contrast to NF-Y, a CCAAT-binding TF that is bound before and after differentiation. The presence of multiple bands in HBP1 western blots is in line with genomic annotations, showing the presence of various HBP1 isoforms. To verify this, and check the specificity of the anti-HBP1 antibody, we inactivated HBP1 in primary keratinocytes by siRNA. Supplementary Figure S2, panel B indicates that all bands, except one, are substantially decreased in extracts of cells transfected with HBP1 siRNA with respect to scramble siRNA. These bands are largely generated by alternative splicing (S Borrelli and R. Mantovani, in preparation). Finally, immunofluorescence assays with HBP1 and p63 antibodies confirm the rise of HBP1 protein that matches the decrease in p63 staining over 7 days of differentiation (Figure $3 c$ ). Note that the decrease in p63 levels can only be appreciated at late time points, most likely because of the fact that immunofluorescence is not quantitative. Essentially the same result is obtained in the HaCaT cell line over a period of 3 days (not shown).

HBP1 is required for keratinocyte stratification. We next verified the expression of HBP1 in human interfollicular skin by confocal immunfluorescence using antibodies against $p 63$ 
and HBP1. Figure 4a shows that p63 decorates basal cells and progressively fades in the granular and spinous layers; in contrast, HBP1 shows a weak expression in the lower layers, whereas it is strongly expressed in the cells of the granular layer, in which there is some overlap with cells expressing low levels of p63. Most of the expression, however, is confined to the subcorneum cells, which are p63 negative.
The staining of the two proteins is consistent with a negative effect of high p63 levels on the expression of HBP1. These results further confirm the reciprocal expression between p63 and HBP1, and suggest that HBP1 might be involved in differentiation of keratinocytes. To ascertain this, we functionally inactivated HBP1 by RNAi in NHEKs before and after differentiation, and compared the expression of
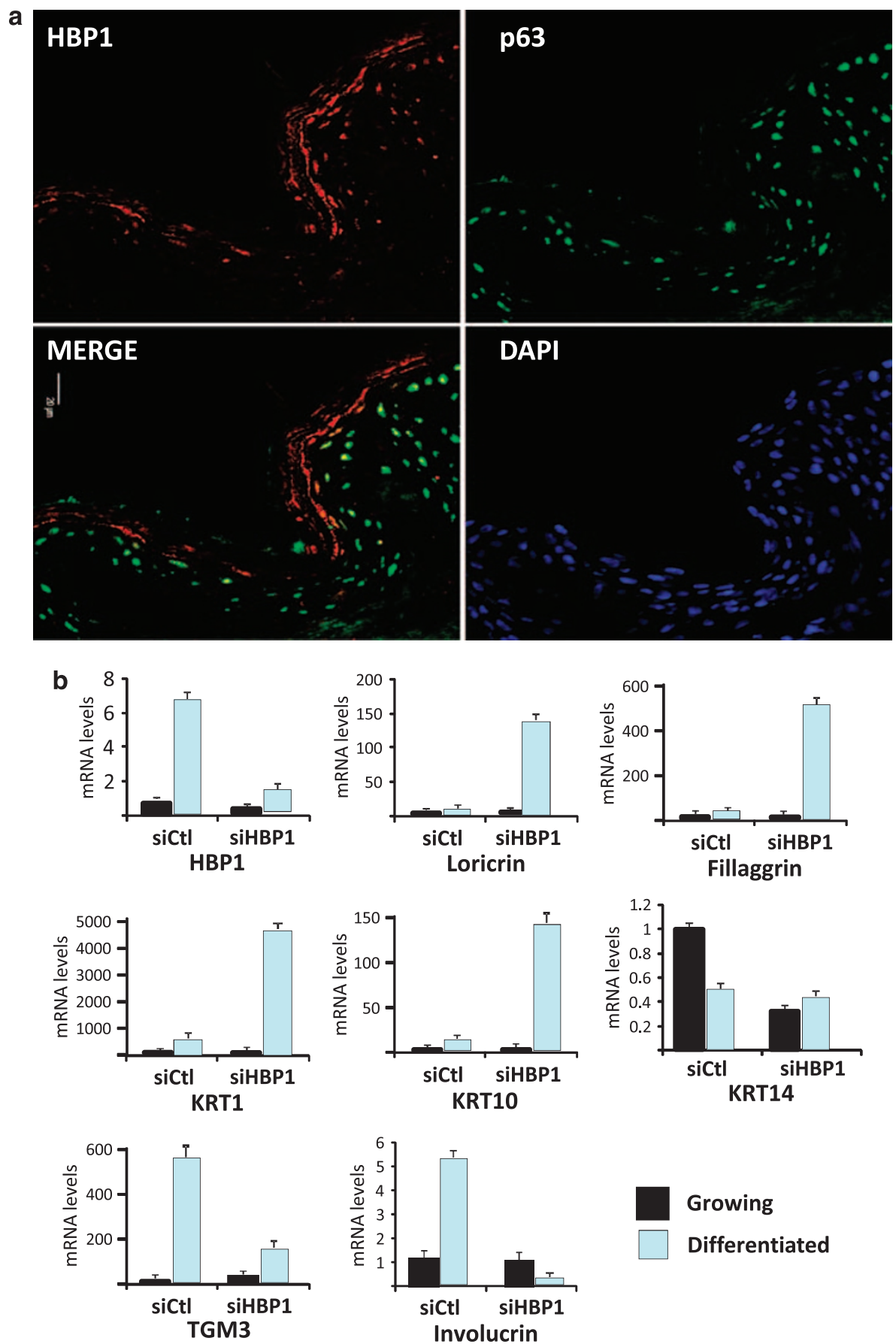

Figure 4 HBP1 is important for keratinocyte differentiation. (a) Confocal immunofluorescence analysis of human interfollicular skin stained with anti-p63 and anti-HBP1 antibodies. (b) Quantification of differentiation markers by qRT-PCR of HBP1-inactivated cells induced to differentiate. NHEKs were transfected with scramble and HBP1 siRNAs. After $24 \mathrm{~h}$, half of the cells were induced to differentiate by addition of calcium. Samples were harvested after 7 days, RNA prepared and qPCR performed on the indicated genes. Values are normalized to GAPDH, used as an internal control 
siCTL

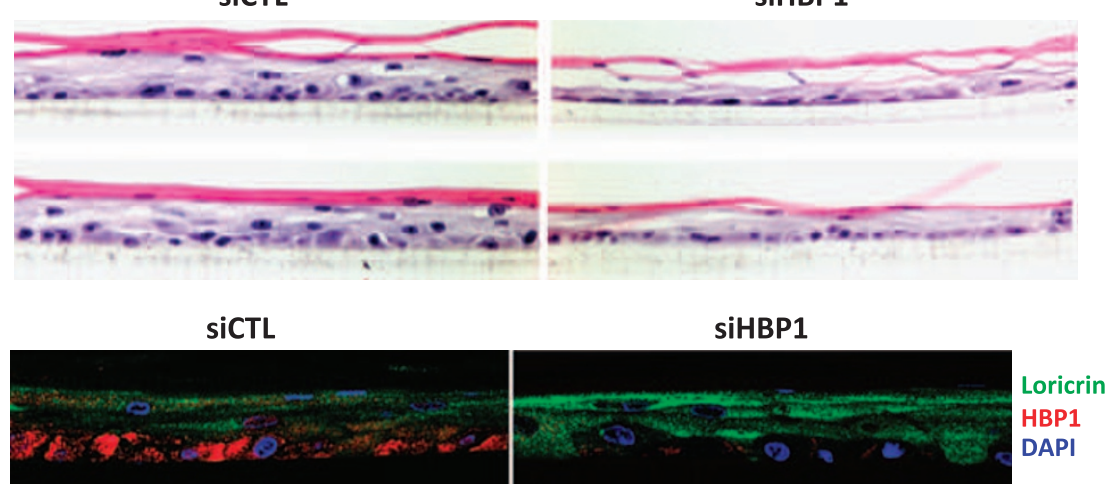

Figure 5 HBP1 is important for skin stratification. H\&E staining of skin 3D reconstitution assays after 7 days of stratification. Human primary keratinocytes were cultured on filter, transfected with siCTL (upper left panel) and siHBP1(upper right panel) and then exposed to 3D conditions. Samples were collected from two wells for each condition (upper and lower panels in each condition). (Lower panels) Immunofluorescence analyses of HBP1 and loricrin expression in 3D reconstituted skin transfected with siCTL (left) and siHBP1(right)

differentiation markers in cells transfected with scramble siRNA. Figure 4b shows the qRT-PCR analysis; a substantial decrease in involucrin and TGM3 was obtained by HBP1 siRNA treatment, particularly after differentiation. On the other hand, the levels of the basal KRT14 drop after differentiation, but no further decrease in HBP1 siRNAtreated NHEKs was observed, indicating that this basal marker is not under the control of HBP1. An opposite effect was observed on other markers of differentiation, such as loricrin, filaggrin and KRT1 and KRT10. All these genes, which are induced during differentiation, show a further increase in their expression in silenced cells. These data suggest that HBP1 inactivation affects differentiation of human keratinocytes, exerting a complex influence, positive and negative, on markers of the upper layers.

To confirm the importance of HBP1 in skin homeostasis, we performed a tri-dimensional organotypic skin reconstitution assay with NHEKs transfected with siRNA of HBP1 and Ctl siRNAs. As shown in Figure 5, tissues regenerated from keratinocytes treated with siHBP1 show impaired stratification compared with siCtl (upper panels for hematoxylin and eosin stainings). We performed immunofluorescence analysis of loricrin and observed a significant increase in its expression in epidermis silenced of HBP1 (Figure 5, lower panels), in agreement with the qPCR analysis performed in Figure $4 \mathrm{~b}$. As expected, loricrin localization in normal human skin sections shows positive staining only in the subcorneum stratum (S Borrelli and R Mantovani, unpublished data). These data indicate that HBP1 has a key role in the stratification of human skin in organotypic cultures.

Characterization of the HBP1 transcriptome in keratinocytes. Only a handful of genes controlled by HBP1 are known, and the complex effects exerted by removal of HBP1 prompted us to evaluate in an unbiased way the HBP1-dependent transcriptome in keratinocytes. We profiled gene expression of siRNA-inactivated cells. Before that, we performed two types of controls. First, we transfected NHEK cells with siRNA for HBP1 and with a vector coding for HBP1 in parallel. We also controlled Wnt signaling targets previously known to be regulated by HBP1. Figure $6 a$ shows
qRT-PCR of c-MYC, c-Jun, cyclin D1 and Sost-DC1 levels: all mRNAs are increased upon HBP1 inactivation, and decreased, with different kinetics, after HBP1 overexpression. Second, we assessed direct HBP1 binding in vivo in ChIP assays. Figure $6 \mathrm{~b}$ indicates that HBP1 is bound to the C-Jun and cyclin D1 promoters in an area in which potential binding sites are located. Therefore, the alteration of HBP1 levels in keratinocytes directly affects expression of known targets of HBP1.

For profiling, we decided to use the RNAi approach, which is potentially less prone to indirect effects because of the vast overexpression of a TF. To minimize individual variations, we collected primary keratinocytes from three healthy donors and independently transfected them with control and HBP1 siRNA. The levels of HBP1 in these cells were low, yet readily detectable. After evaluation of the levels of HBP1 inactivation, which were $>80 \%$ (not shown, see Supplementary Figure S2, panel C), samples with an equal amount of RNA were pooled and profiling was performed on the Illumina platform in triplicate. Considering a threshold of 1.35 over control, 272 genes were upregulated and 48 were downregulated. A list of the regulated genes is shown in Supplementary Figure S3. We validated some of the identified genes by independent RNAi experiments; most showed the expected variation in qRT-PCR analysis (Figure 7). We noticed that the changes in gene expression measured by qRT-PCR are slightly greater than those recorded by the Illumina expression profiling platform, suggesting that additional genes might have been missed because of the relatively stringent threshold imposed to the analysis. We next performed Gene Ontology (GO) analysis on the targeted genes, considering terms with $P$-values $<10^{-6}$ (Figure 8 ). In the biological processes, negative regulation of proliferation and terms related to development and differentiation predominate; in the cellular component and molecular function analysis, extracellular space and secretory functions are enriched. Specifically, many downregulated genes code for proteins that are involved in barrier function in the upper layers of the skin (see Discussion). This analysis corroborates our findings that HBP1 is an important and complex regulator of the differentiation program in human keratinocytes. 
a

c-MYC

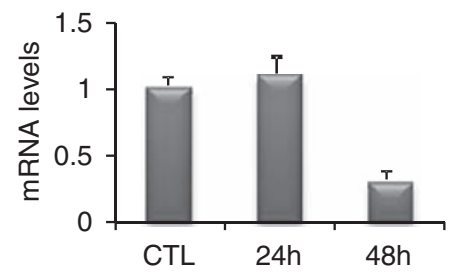

HBP1 overexpression

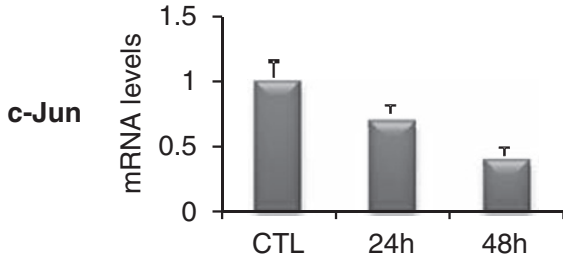

HBP1 overexpression

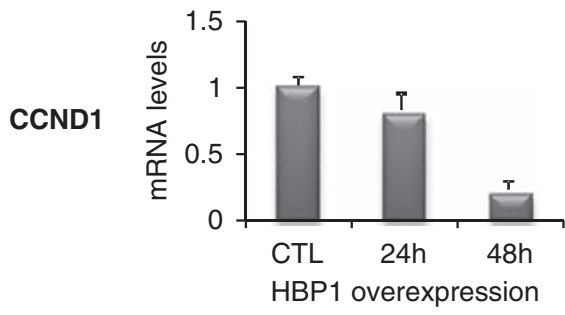

SOST-DC1

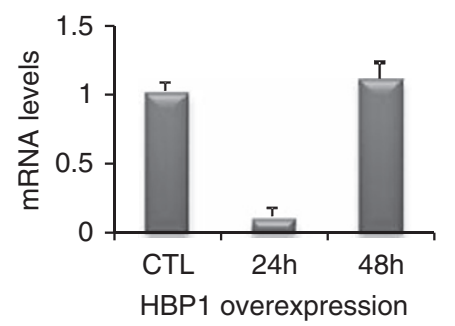

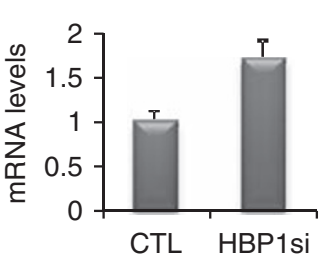

HBP1 knock down

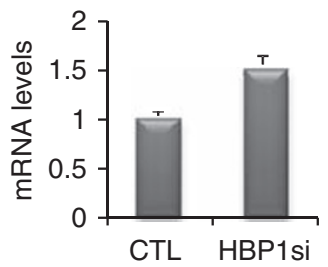

HBP1 knock down
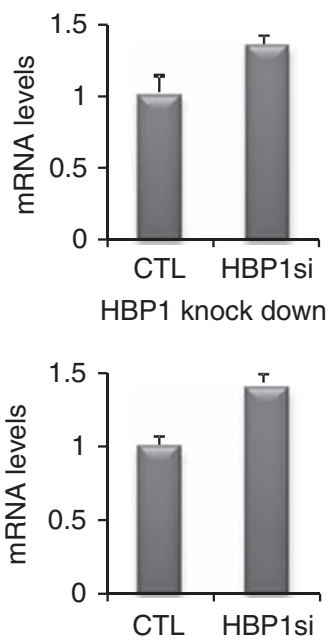

HBP1 knock down

b

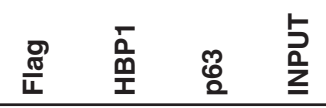

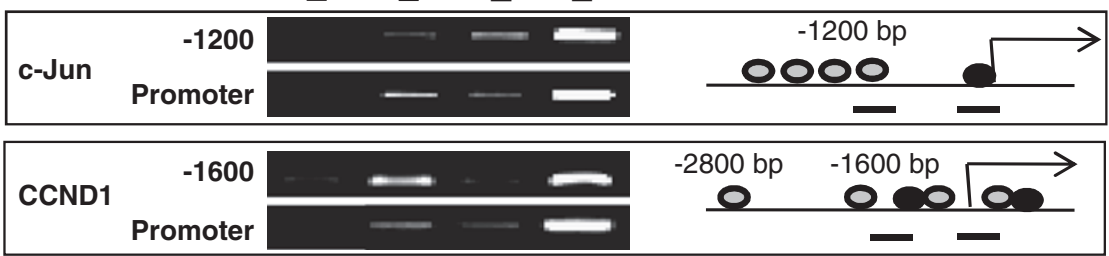

Figure 6 HBP1 directly regulates Wnt genes in NHEKs. (a) Expression of genes of the Wnt pathway in NHEK in which HBP1 levels are altered, positively or negatively. In the left panels, qRT-PCR of c-Myc, c-Jun, cyclin D1 and Sost-DC1 upon overexpression of HBP1 for 24 and $48 \mathrm{~h}$. In the right panels, after HBP1 RNAi inactivation at $48 \mathrm{~h}$ after transfection. (b) In vivo binding of HBP1. Chromatin immunoprecipitation of NHEK cells with $\alpha$-HBP1 and control $\alpha$-Flag antibodies. Amplicons in the c-Jun and cyclin D1 promoters are used to evaluate the specific enrichments of HBP1 relative to the control antibody. Right panels show a schematic representation of the c-Jun and cyclin D1 promoter loci, with black and gray dots indicating, respectively, in silico HBP1 and p63 sites in the appropriate position. The black bars indicate the amplicons used in the ChIP experiments

\section{Discussion}

In this report, we detail the connections between p63 and HBP1 in keratinocytes. Originally emerging from an RNAi profiling screening, HBP1 has been confirmed as a direct target of $\mathrm{p} 63$ by several criteria, in vitro and in vivo. HBP1 is important for skin differentiation, and profiling analysis confirms that it partakes in a program of advanced differentiation and stratification.

p63 inhibits a potent growth suppressor. The key to our understanding of the strategy of TFs in regulating cellular functions is the identification of targets. The p63 network in keratinocytes was identified by two strategies: gene 


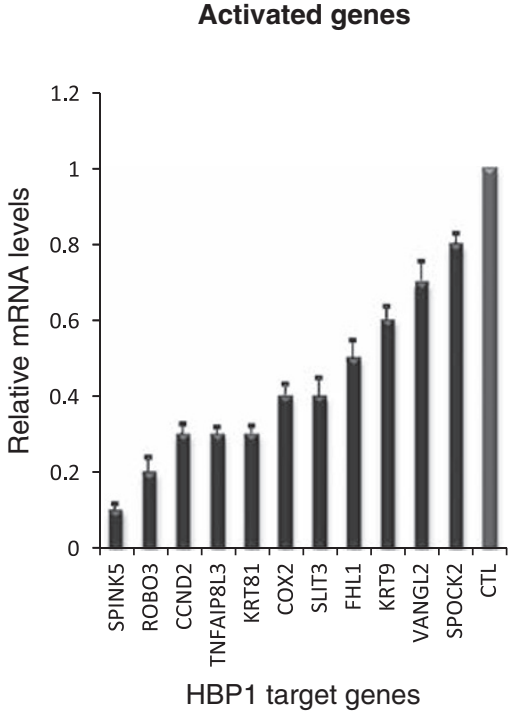

Activated genes

HBP1 target genes

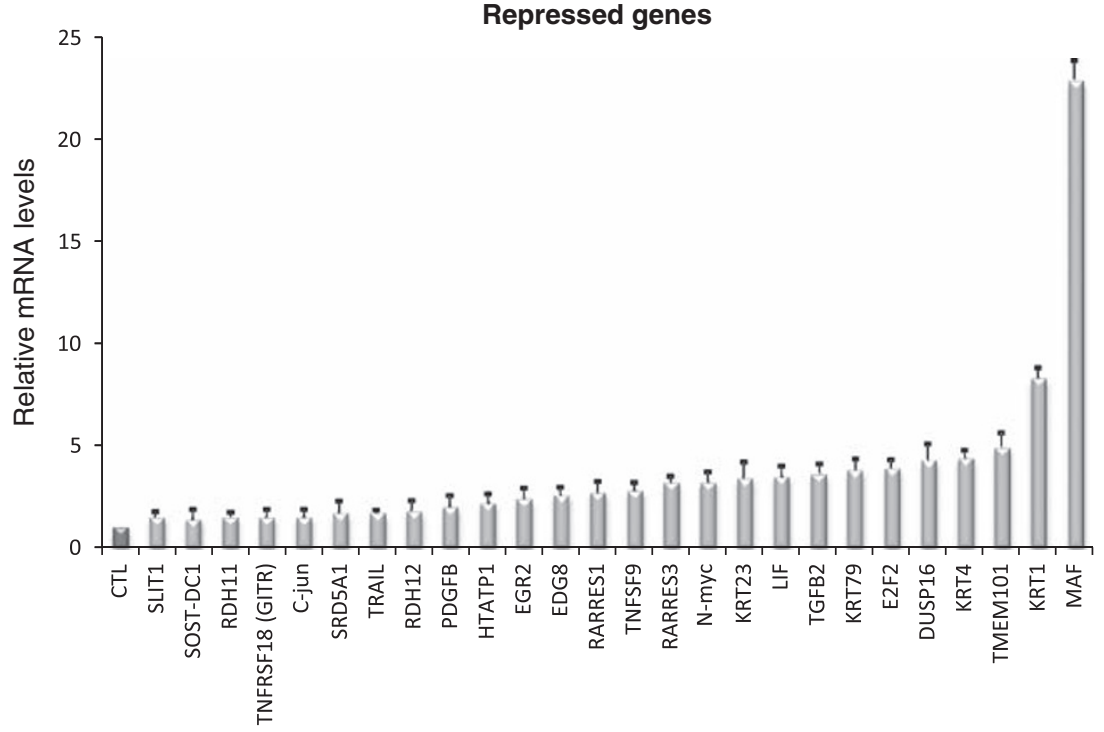

HBP1 target genes

Figure 7 Validation of gene expression profiling. q-RTR-PCR analysis of HBP1-activated and HBP1-repressed targets that emerged from the expression profiling of HBP1-inactivated NHEKs. The values of the indicated mRNAs in NHEKs in which HBP1 was inactivated are plotted with those of NHEKs transfected with a control scramble siRNA

expression profiling of p63-depleted cells ${ }^{10}$ and ChIP on chip. Genes involved in growth, adhesion and transcriptional regulation are the most significant GO terms enriched.

Essentially, TFs involved in all pathways known to have a role in differentiation - Notch, TGF $\beta, \mathrm{Wnt} / \beta$-catenin, nuclear receptors, $\mathrm{SH}$ and $\mathrm{C} / \mathrm{EBPs}$ - are under direct $\mathrm{p} 63$ regulation. A network of reciprocal interplay is emerging, which fine tunes and adapts the relative levels of the TFs involved to the various external conditions. p63 has well-established pro-growth activities that regulate stem cell potential of pluristratified epithelia; in addition, it is involved in the establishment of the stratification program in the embryo, but it is currently debated whether it partakes in all phases of differentiation, including cornification. Simple considerations concerning the levels of p63 expression in the different skin layers suggest that it is involved in the growth of proliferating and early differentiating lower layers of epidermis. As these cells mature, other TFs are predominantly expressed, with essentially two missions: promote expression of differentiation genes eventually involved in barrier formation, and exit from the cell cycle. IRF6, Ikk $\alpha$, Gata3 and C/EBP $\delta$ are p63 targets with these effects, ${ }^{8,11-13}$ and our data indicate that HBP1 joins this list, as determined by ChIP, RNAi, overexpression, transient luciferase assays and evaluation of HBP1 levels in wt, p63 KO and transgenics. It is worth noting that in this latter context, HBP1 levels are mainly controlled by the levels of p63, whereas other p63 targets, such as EGF-R, show independence from p63 levels, suggesting that they are controlled by other important TFs. The strong and dominant repressive effect of $\mathrm{p} 63$ on $\mathrm{HBP} 1$ is consistent with the growth-suppressive role of the latter. In this scenario, p63 would have to downplay all other TFs that interfere with growth and promote terminal differentiation. Interestingly, unlike many other TFs, inactivation of HBP1 leads to essentially no alteration in the levels of p63, indeed suggesting that the latter is acting upstream of HBP1 in the differentiation cascade, and that p63 downregulation, which is observed in the upper layers, is exerted through different mechanisms.

HBP1 in keratinocyte differentiation and stratification. The discovery of HBP1 was followed by several papers reporting that the gene is induced in many differentiation systems, including muscle, hematopoietic cells and adipocytes. The process takes a few days to complete; whenever measured, the kinetics of induction of HBP1 were relatively fast - within a few hours - and lasted 12-18 h maximum, consistent with a relatively early role in the process. In muscle cells, inactivation of HBP1 by siRNA was matched by lack, or reduced, differentiation. The repressive activities of HBP1 are believed to be responsible for the repression of $\mathrm{G}_{1} / \mathrm{S}$ genes by recruitment of $\mathrm{Rb}$ and pocket proteins, and their associated histone-modifying complexes. ${ }^{4}$ However, our ChIP data are the first direct evidence that this mechanism is operational in vivo through direct sequencespecific promoter interactions. Furthermore, the capacity to interfere with key genes activated by Wnt signaling, regulated through TCFs, induces withdrawal from the cell cycle and differentiation. The keratinocytes data are consistent with these observations, in terms of kinetics of induction (Figure 3), expression levels in suprabasal layers of human and mouse skin (Figures 2 and 4) and requirement of HBP1 to complete the process of differentiation and stratification (Figure 5). The vastly impaired stratification of skin sections inactivated of HBP1 is currently the first example of the importance of HBP1 for tissue differentiation. The role of HBP1 is evidently complex, as some markers of differentiation, such as TGM3 and involucrin, are reduced, whereas loricrin is increased, both in the primary culture and in 


\begin{tabular}{|c|c|c|}
\hline BIOLOGICAL PROCESS & p.val & ANALYZED GENES \\
\hline negative regulation of epithelial cell proliferation & $2.17 \mathrm{e}-10$ & KRT4 RARRES1 RARRES3 TGFB2 \\
\hline tissue development & $2.17 \mathrm{e}-06$ & KRT1 KRT9 TGFB2 SPINK5 \\
\hline regulation of epithelial cell proliferation & $7.92 \mathrm{e}-09$ & KRT4 TGFB2 \\
\hline epithelial cell proliferation & $2.73 e-07$ & KRT4 TGFB2 \\
\hline epidermis development & $3.58 \mathrm{e}-06$ & KRT1 KRT9 TGFB2 SPINK5 \\
\hline negative regulation of cell proliferation & $3.8 \mathrm{e}-06$ & KRT4 RARRES1 RARRES3 TGFB2 \\
\hline ectoderm development & $6.38 \mathrm{e}-06$ & KRT1 KRT9 TGFB2 SPINK5 \\
\hline extracellular structure organization and biogenesis & $3.17 e-07$ & TGFB2 SPOCK2 SPINK5 \\
\hline extracellular matrix organization and biogenesis & $1.16 \mathrm{e}-06$ & TGFB2 SPOCK2 SPINK5 \\
\hline chemotaxis & $3.2 \mathrm{e}-06$ & PDGFB TGFB2 ROBO3 \\
\hline axon guidance & $4.58 \mathrm{e}-06$ & SLIT1 SLIT3 TGFB2 ROBO3 \\
\hline hormone metabolic process & $6.99 \mathrm{e}-06$ & SRD5A1 RDH11 RDH12 \\
\hline behavior & $7.18 \mathrm{e}-06$ & EGR2 PDGFB TGFB2 ROBO3 \\
\hline cell motility & $7.75 \mathrm{e}-06$ & PDGFB SLIT1 SLIT3 TGFB2 ROBO3 \\
\hline phosphate transport & $8.11 \mathrm{e}-06$ & \\
\hline defense response to bacterium & $9.38 \mathrm{e}-06$ & \\
\hline \multicolumn{3}{|l|}{ CELLULAR COMPONENT } \\
\hline extracellular space & $2.17 \mathrm{e}-10$ & SLIT1 SLIT3 TNFSF10 TNFSF9 LIF \\
\hline secretory granule & $1.52 \mathrm{e}-07$ & PDGFB TGFB2 SPINK5 \\
\hline cytoplasmic membrane-bounded vesicle lumen & $3.27 e-07$ & PDGFB TGFB2 \\
\hline extracellular matrix & $7.02 \mathrm{e}-07$ & SPOCK2 \\
\hline intermediate filament cytoskeleton & $1.29 \mathrm{e}-06$ & $\begin{array}{l}\text { KRTI KRT4 KRT9 KRI81 KRTLS } \\
\text { KRT79 }\end{array}$ \\
\hline basement membrane & $3.26 \mathrm{e}-06$ & \\
\hline extracellular matrix part & $4.73 e-06$ & \\
\hline \multicolumn{3}{|l|}{ MOLECULAR FUNCTION } \\
\hline extracellular matrix structural constituent & $2.85 \mathrm{e}-06$ & \\
\hline serine-type endopeptidase inhibitor activity & $6.99 \mathrm{e}-06$ & SPINK5 \\
\hline endopeptidase inhibitor activity & $1.53 e-05$ & SPINK5 \\
\hline enzyme inhibitor activity & $2.57 \mathrm{e}-05$ & SPINK5 \\
\hline endopeptidase activity & $7.59 \mathrm{e}-05$ & \\
\hline cytokine activity & $8.91 \mathrm{e}-05$ & PDGFB TGFB2 TNFSF10 TNFSF9 \\
\hline
\end{tabular}

Figure 8 Gene ontology categorization of HBP1-regulated genes in NHEKs. Gene ontology analysis of HBP1 targets. For the categorization of biological processes, we considered terms with $P$-values $<10^{-6}$, and for cellular components and molecular function, terms with $P$-values $<10^{-5}$

the tri-dimensional systems, and hence the need for transcriptome analysis.

The list of HBP1-regulated genes is very informative of the role of HBP1 in keratinocytes (Figure 8). Enriched GO terms, such as regulation of epithelial differentiation, epidermis development and regulation of epithelial proliferation, were expected and they are reassuringly at the top of the list. We found fewer genes whose expression was decreased after RNAi than genes that were increased, confirming that this TF is primarily a repressor. However, HBP1-activated genes exist and many of them do have a role in differentiation (Figure 9). One of these is SPRRs, a member of a large cluster of genes whose expression is coordinated. ${ }^{14}$ Small prolinerich proteins are primary constituents of the stratum corneum (SC), expressed in the granular layer, with no staining in the spinous or basal layers. SPINK proteins are serine protease inhibitors of the Kazal type, also involved in skin barrier formation. In fact, mutations in the Spink5 (LEKTI) gene cause the Netherton syndrome, characterized by ichthyosiform dermatosis and hair shaft abnormalities, ${ }^{15}$ and Spink5 KO mice have severe alteration in the SC. ${ }^{16}$ Additional activated targets are COX-2, FLH1 and ROBO3. COX-2 is expressed in the upper layer and is involved in differentiation. ${ }^{17} \mathrm{FHL} 1$, also known as KyoT1/2 or SLIM1, is a LIM domain transcriptional co-activator whose expression suppresses differentiation; it modulates Notch and TGF $\beta$ signaling through interactions with RBP-J and SMADs, respectively. ${ }^{18,19}$ Thus, the link with this TF could expand the consequences of HBP1 modulation to two important pathways, which are also affected directly by p63. ${ }^{20}$ Opposite regulation is observed for the Spink1-Furin complex and for other Spink targets, the Kallikreins-related peptidases (KLKs); specifically, Furin is a subtilysin-like convertase abundantly expressed in epidermal keratinocytes and involved in differentiation, ${ }^{21}$ and KLK4 and KLK14 are serine proteases implicated in the desquamation process of the skin. ${ }^{22}$ TGM5 is a transglutaminase, whose mutation is 


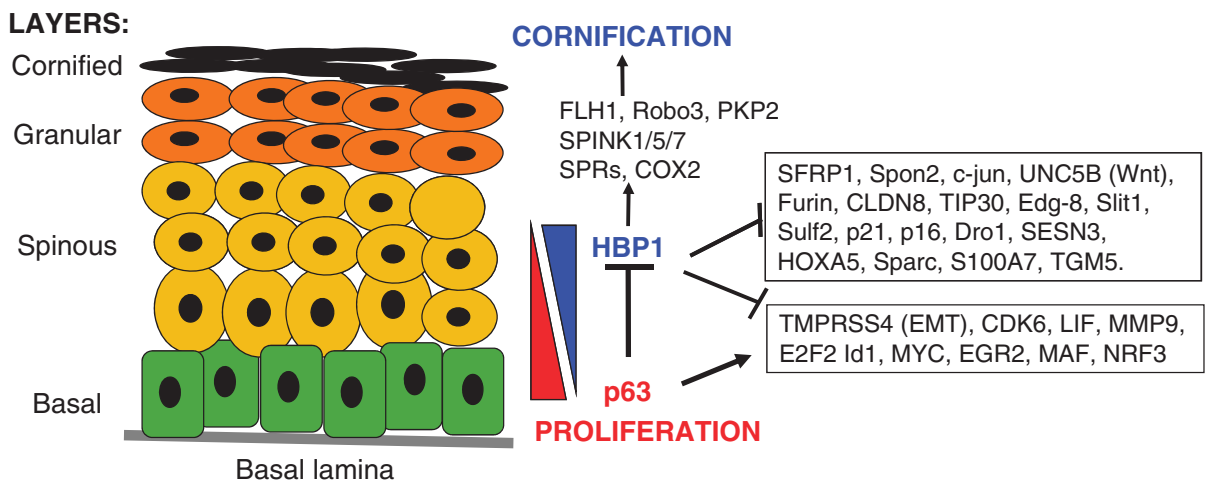

Figure 9 Scheme of expression of HBP1 targets in human skin

associated with the Acral peeling skin syndrome. ${ }^{23}$ Thus, fine tuning of proteases and inhibitors in upper layers is apparently a major task of HBP1. Additional coregulation is observed for $\mathrm{ROBO}$, a transmembrane receptor, and for one of its ligands, SLIT1. ${ }^{24}$

As for repressed genes, there are several genes of the Wnt pathway; in addition to c-Jun and c-Myc, SFRP1, SPON2, UNC5B, PKP2 and COX2 are new targets. ${ }^{25-28}$ Recent data reported in breast cancer indicate that HBP1 is structurally altered, through poorly identified mechanisms, in $16 \%$ of tumors, and specifically silenced in the majority of aggressive tumors. ${ }^{7}$ Indeed, the lack of HBP1 expression is of high prognostic value, together with that of SFRP1, as markers of relapse and poor prognosis. SFRP1 is a negative regulator of Wnt signaling: the notion that HBP1 controls SFRP1 levels is consistent with a tumor-suppressive function of the former. In this respect, it will be interesting to ascertain whether a direct functional link is present in breast cells as well, and whether HBP1 and SFRP1 levels are also reduced in metastatic skin cancers. COX-2 is another gene whose altered levels are associated with breast malignancies, ${ }^{28}$ and Paulson et al. ${ }^{7}$ indeed hypothesized that COX-2 overexpression is an indirect effect of HBP1 inactivation. In line with a growth-suppressive function of HBP1 is the repression of a number of growthpromoting genes, such as PDGF $\beta 2$, CDK6, E2F2, Id1, EGR2/KROX20, HOXA5, and SPARC, all expressed in lower skin layers, ${ }^{29-33}$ or LIF, expressed in the granular layer, ${ }^{34}$ as well as MMP9, a methalloprotease involved in metastasis, and TMPRSS4, involved in epithelial mesenchimal transition (EMT). ${ }^{35}$ Finally, although there are no data on TIP30 in the skin, overexpression of this gene stimulates, and inactivation represses, metastasis of prostate cancer cells. ${ }^{36}$

Intriguingly, there are two groups of genes whose repression is apparently at odd with a simplistic model of HBP1 as an antigrowth, pro-differentiation TF. The first relates to several repressed genes specifically expressed in the upper layers: KRT1 and KRT4, as well as KRT9, a location-specific cytokeratin expressed in palmoplantar epidermis; ${ }^{37,38}$ loricrin and filaggrin, terminal differentiation markers, and S100A7 (psoriasin), which is expressed in the superficial, differentiated cells and whose expression is altered in psoriatic skin. ${ }^{39}$ SLIT1 and RARRES1-3 (also known as TIG1-3) are also involved in differentiation. ${ }^{40}$ Claudin- 8 is a component of tight junctions. ${ }^{41}$ It is possible that HBP1 repression of these genes is a matter of proper timing during terminal differentiation, and that other TFs are directly involved in relieving the early HBP1repression after cell-cycle block to activate this class of genes.

The second is a group of genes that are notoriously associated with growth suppression in keratinocytes and other cell types: SPRX/Dro1, ${ }^{42}$ the p53-targeted antioxidants SESN3 and SULF2 ${ }^{43,44}$ and Edg-8. ${ }^{45}$ p21 and p16INK4 are also in this class. The former was previously identified as a target; ${ }^{46}$ a possible explanation as to the significance of this class comes from inactivation of p16INK4, which allows primary keratinocytes to escape replicative senescence, apparently maintaining keratinocytes in the stem cell compartment. ${ }^{47}$ p16INK4 downregulation in transient amplifying keratinocytes, instead, has no effects. Thus, p16INK4 seems to control epidermal stem cells. Could this point to a role of HBP1 in restricting growth in basal layers stem cells, as it has been proposed for $\operatorname{C/EBP} \delta$ in corneal epithelia? ${ }^{12}$ Interestingly, a recent study has identified p16INK4 as a gene repressed by $p 63$ in vivo, in elegant rescue experiments with $\mathrm{KO}$ mice. ${ }^{48}$ Our data, showing repression of p16INK4 by HBP1, suggest that this growth suppressor might be the focus of important convergent signals between the two TFs. In general, the cohort of genes that are under mutual control is quite significant, with variation depending on the source of p63 targets considered (summarized in Supplementary Figure S4); overall, 80 out of 320 genes regulated by HBP1 are found in one or more lists of p63 targets.

Finally, two targeted TFs seem to have important regulatory roles during development, which might be related to p63 function. (1) Strong HOXA4 expression is detected in the basal layer of day 10 mice fetal epidermis; ${ }^{32}$ incidentally, this timing correlates with the onset of p63 expression and with epidermal stratification. In newborn and adult life, expression of HOXA4 moves up to the granular layer of newborn and adult mice skin, suggesting that this TF might be important for the initial stratification program and for further differentiation in adult life. (2) The leucine zipper c-maf is first expressed on embryonic day 16 in the basal layer of epidermis, increases until day 19 and by postnatal day 3 expression disappears, ${ }^{49}$ suggesting that it might be involved in the maintenance of proliferative potential in the embryonic period.

In summary, our work identifies HBP1 as an important hub in decision making during keratinocyte differentiation that is targeted negatively by p63; it also defines two classes of genes involved in terminal differentiation that are differentially regulated by HBP1. Future studies on the network of HBP1 
targets should aim at clarifying their role in normal conditions and neoplastic transformation, as the network of regulated genes might be altered in expression in specific tumors.

\section{Materials and Methods \\ Cells and culture conditions. HaCaT cells were grown in DMEM in the presence of $10 \%$ fetal calf serum. First passage primary NHEKs were derived from breast of healthy individuals and grown on a feeder layer of lethally irradiated 3T3 cells in DMEM/F12 added of insulin $(5 \mu \mathrm{g} / \mathrm{ml})$, EGF-R $(10 \mathrm{ng} / \mathrm{ml})$ hydrocortisone $(0.4 \mu \mathrm{g} / \mathrm{ml})$, T3 $(2 \mathrm{nM})$, cholera toxin $(0.1 \mathrm{nM})$ and transferrin $(5 \mu \mathrm{g} / \mathrm{ml})$. NHEKs were differentiated by adding $\mathrm{CaCl}_{2}$ (1.4 mM final concentration) in the presence of $10 \%$ fetal calf serum, and $\mathrm{HaCaT}$ cells were differentiated by adding calcium and lowering FCS to $0.1 \%$.}

3D human keratinocyte culture. The culture is based on Millicell hanging cell culture insert (polyethylene terephthalate, $1.0 \mu \mathrm{m}$, Millipore, Billerica, MA, USA). Early passage (passage 2) human keratinocytes were seeded at a density of $50000 /$ insert in $0.2 \mathrm{ml}$ expansion medium (CNT-07; CELLnTEC, Berne, Switzerland). The inserts were then placed in a 12-well plate with $1 \mathrm{ml}$ CNT-07 medium added into the well. The culture was maintained for $8 \mathrm{~h}$ at $37^{\circ} \mathrm{C}, 5 \% \mathrm{CO}_{2}$ before siRNA transfection.

RT-PCR, $\mathbf{q}-\mathbf{P C R}$ and transfections. HaCaT cells were transiently transfected using Oligofectamine (Gibco-BRL, Dorchester, Dorset, UK) for $3 \mathrm{~h}$ with $150 \mathrm{ng} / \mathrm{cm}^{2}$ of human $\mathrm{p} 63$ siRNA oligonucleotide that targets the central DNAbinding domain of $p 63 .^{8}$ After overnight incubation, transfection was repeated for an additional $3 \mathrm{~h}$. First passage NHEKs, $2.5 \times 10^{5}$, were transfected with Nucleofector (Amaxa, Lonza AG, Cologne, D) using siRNA ologonucleotides at $0.5 \mathrm{nM}$. p63 inactivation was described in Chikh et al. ${ }^{8}$ and Jenkins et al. ${ }^{37}$ The HBP1 siRNA was $5^{\prime}$-GCUUGACUGUGGUACAGCATT- $3^{\prime}$ and $5^{\prime}$-UGCUGUACCACAGUCAAGCTT-3' (Ambion, Austin, TX, USA); an off-target siRNA oligos mixture (5'-AUGAACGUGAA UUGCUCAA-3', 5'-UAAGGCUAUGAAGAGAUAC-3', 5'-AUGUAUUGGCCUGUA UUAG-3' and 5'-UAGCGACUAAACACAUCAA-3'; Dharmacon D-00181001 (Thermo Fisher Scientific Inc., Waltham, MA, USA)) was used as control. RNA was extracted with RNA-Easy kit (Qiagen, Hilden, Germany) $48 \mathrm{~h}$ after siRNA transfections. For CDNA synthesis, $4 \mu \mathrm{g}$ of RNA was retrotranscribed with M-MLVRT kit (Invitrogen, Carlsbad, CA, USA). Semiquantitative PCR analysis was performed with primers listed in Supplementary Figure S5. For q-PCR, a Bio-Rad MylQ single-color thermal cycler (Hercules, CA, USA) and a SYBR Green PCR Master mix (Genespin, Milan, Italy) were used. Specificity of products was monitored with a heat dissociation curve. Fold changes of expression were calculated with the formula $2-\Delta \mathrm{Ct}$ where $\mathrm{Ct}$ represented the threshold cycles of the specific gene and GAPDH. The HBP1 promoter $(-1200$ to +50$)$ was PCR amplified with PFU polymerase (Genespin, Milan, Italy) and cloned into Xhol Bgll sites of pGL3-basicluciferase. For transactivation experiments, $1 \times 10^{5} \mathrm{NHEK}$ cells were transfected with Lipofectamine (Gibco-BRL) using $1.2 \mu \mathrm{g}$ of HBP1-luciferase reporter plasmid, $200 \mathrm{ng}$ of p63 different splicing isoforms and carrier for a total DNA of $2 \mu \mathrm{g}$. Four independent transfections in duplicate were performed.

RNAi in 3D system. For siRNA transfection, for each well, 120 pmole of siRNA duplexes and controls was diluted into $200 \mu \mathrm{l}$ of CNT-07 medium, and was then mixed with $10 \mu \mathrm{l}$ of INTERFERin (Polyplus, Illkirch, France) for $10 \mathrm{~min}$ at room temperature. Then, $200 \mu \mathrm{l}$ of transfection mix was added into the inserts to replace the previous medium in it. The achieved final siRNA concentration was $100 \mathrm{nM}$. The cells were first grown in CNT-07 medium for $48-72 \mathrm{~h}$ till $100 \%$ confluence and then maintained into 3D medium (CNT-02-3DP5, CELLnTEC). After $16 \mathrm{~h}$, the medium in the insert was aspired and the cells were exposed to air. In the meantime, the medium in the wells was refreshed. The $3 \mathrm{D}$ culture was then maintained for 7 days by changing the medium every day. After that the samples were collected for immunohistological analysis and RNA extraction. All the samples were performed at duplex.

Chromatin immunoprecipitations. ChIP analyses were carried out as previously described by Grose et al. ${ }^{29}$, with $5 \mu \mathrm{g}$ of anti-p63 antibody (Genespin, I), $5 \mu \mathrm{g}$ of HBP1 antibody (Active Motif, Carlsbad, CA, USA) and $5 \mu \mathrm{g}$ of Flag control antibody (Sigma, St Louis, MO, USA). The list of primers used in the PCR amplifications is summarized in Supplementary Figure S5.

Immunofluorescence and Immunohistochemistry. Immunofluorescence analysis was performed as in Grose et al. ${ }^{29}$ with the following antibodies: p63
(Genespin, I), HBP1 (Active Motif) and KRT14 (Neomarkers, Labvision, Fremont, CA, USA). For IHC, $14 \mu \mathrm{M}$ paraffin sections were deparaffined and then blocked for $60 \mathrm{~min}$ in PBST ( $0.1 \%$ Triton $\times 100$ in PBS) with $2.5 \%$ donkey serum, $2.5 \%$ goat serum, $0.5 \%$ cold water fish gelatin and $0.5 \%$ BSA. Primary antibodies were applied for $16 \mathrm{~h}$ at $4{ }^{\circ} \mathrm{C}$ and secondary antibodies were applied for $1 \mathrm{~h}$ at room temperature. DAPI nuclei counterstaining was used.

RNA profiling. NHEK cells from three independent healthy donors were transfected by Lipofectamine (Gibco-BRL) with scramble and HBP1 siRNA. Cells were harvested after $48 \mathrm{~h}$ and RNA prepared with the RNA-Easy kit. RNA concentrations were determined using a Nanodrop (NanoDrop, Wilmington, DE, USA) spectrophotometer and quality was assessed using an Agilent 2100 Bioanalyzer (Agilent Technologies, Santa Clara, CA, USA). A total of $2 \mu \mathrm{g}$ of each sample was pooled for further processing. For each sample, $500 \mathrm{ng}$ of total RNA was synthesized to biotinylated cRNA using the lllumina RNA Amplification Kit (Ambion). Synthesis was carried out according to the manufacturer's instructions. cRNA concentration and the quality were assessed out as described above. From each sample, technical replicates were produced and $750 \mathrm{ng}$ CRNA was hybridized for $18 \mathrm{~h}$ to HumanHT12 v. 3.0 Expression BeadChips (Illumina Inc., San Diego, CA, USA) according to the protocol provided by the manufacturer. Hybridized chips were washed and stained with streptavidin-conjugated Cy3 (GE Healthcare, Waukesha, WI, USA). BeadChips were dried and scanned with an Illumina BeadArray Reader (lllumina Inc.).

For data analysis, the intensity files were loaded into the Illumina BeadStudio v. 3.1.3.0 software for quality control and gene expression analysis. First, the quantile normalization algorithm was applied on the data set to correct systematic errors. Background was subtracted. For differential expression analysis, technical replicates of each sample were grouped together and genes with a detection of $P$-value $<0.01$, corresponding to a false-positive rate of $1 \%$, were considered as detected. Differently expressed genes were selected with Diff Score cutoff set at \pm 30 , corresponding to a $P$-value of 0.001 .

\section{Conflict of interest}

The authors declare no conflict of interest.

Acknowledgements. We thank T Kouzarides (Cambridge, UK) for kindly providing the HBP1 plasmids. This work was supported by grants from the European Union (EPISTEM LSHB-CT-2005-019067 to RM, GPD and GM, and CRESCENDO LSHM-CT-2005-018652 to AW), CARIPLO to RM, MIUR (PRIN) and Associazione Italiana per la Ricerca sul Cancro, to RM and AW. SB. has a fellowship from the $\mathrm{U}$ degli Studi di Milano. OMV Grober is a student of the PhD School in Environment, Biological, Biotechnology, Physics, Mathematics and Informatics Sciences/Doctorate in Pathology of Cell Signal Transduction; and M Ravo is a student of the PhD School in Toxicology/Doctorate in Molecular Oncology and Molecular Pathology, University of Cagliari (Italy).

1. Aylon Y, Oren M. Living with p53, dying of p53. Cell 2007; 130: 597-600.

2. McKeon F. p63 and the epithelial stem cell: more than status quo? Genes Dev 2004; 18 : 465-469.

3. Rinne T, Hamel B, van Bokhoven H, Brunner HG. Pattern of p63 mutations and their phenotypes-update. Am J Med Genet A 2006; 140: 1396-1406.

4. Yee AS, Paulson EK, McDevitt MA, Rieger-Christ K, Summerhayes I, Berasi SP et al. The HBP1 transcriptional repressor and the p38 MAP kinase: unlikely partners in $\mathrm{G}_{1}$ regulation and tumor suppression. Gene 2004; 336: 1-13.

5. Yao CJ, Works K, Romagnoli PA, Austin GE. Effects of overexpression of HBP1 upon growth and differentiation of leukemic myeloid cells. Leukemia 2005; 19: 1958-1968.

6. Zhang X, Kim J, Ruthazer R, McDevitt MA, Wazer DE, Paulson KE et al. The HBP1 transcriptional repressor participates in RAS-induced premature senescence. Mol Cell Biol 2006; 26: 8252-8266

7. Paulson KE, Rieger-Christ K, McDevitt MA, Kuperwasser C, Kim J, Unanue VE et al. Alterations of the HBP1 transcriptional repressor are associated with invasive breast cancer. Cancer Res 2007; 67: 6136-6145.

8. Chikh A, Sayan E, Thibaut S, Lena AM, DiGiorgi S, Bernard BA et al. Expression of GATA-3 in epidermis and hair follicle: relationship to p63. Biochem Biophys Res Commun 2007; 361: 1-6.

9. Candi E, Rufini A, Terrinoni A, Dinsdale D, Ranalli M, Paradisi A et al. Differential roles of p63 isoforms in epidermal development: selective genetic complementation in p63 null mice. Cell Death Differ 2006; 13: 1037-1047. 
10. Viganò MA, Mantovani R. Hitting the numbers: the emerging network of p63 targets. Cell Cycle 2007; 6: 233-239.

11. Richardson RJ, Dixon J, Malhotra S, Hardman MJ, Knowles L, Boot-Handford RP et al. Irf6 is a key determinant of the keratinocyte proliferation differentiation switch. Nat Genet 2006; 38: $1329-1334$

12. Barbaro V, Testa A, Di lorio E, Mavilio F, Pellegrini G, De Luca M. C/EBPdelta regulates cell cycle and self-renewal of human limbal stem cells. J Cell Biol 2007; 177 1037-1049.

13. Borrelli S, Testoni B, Callari M, Alotto D, Castagnoli C, Romano R-A et al. Reciprocal regulation of $\mathrm{p63}$ by C/EBPdelta in human keratinocytes. BMC Mol Biol 2007; 8: 85

14. Koizumi $H$, Kartasova $T$, Tanaka $H$, Ohkawara A, Kuroki T. Differentiation-associated localization of small proline-rich protein in normal and diseased human skin. $\mathrm{Br} J$ Dermatol 1996; 134: 686-692.

15. Mägert HJ, Kreutzmann P, Drögemüller K, Ständker L, Adermann K, Walden M et al. The 15-domain serine proteinase inhibitor LEKTI: biochemical properties, genomic organization, and pathophysiological role. Eur J Med Res 2002; 7: 49-56.

16. Yang T, Liang D, Koch PJ, Hohl D, Kheradmand F, Overbeek PA. Epidermal detachment, desmosomal dissociation, and destabilization of corneodesmosin in Spink5-/- mice. Genes Dev 2004; 18: 2354-2358.

17. Leong J, Hughes-Fulford M, Rakhlin N, Habib A, Maclouf J, Goldyne ME. Cyclooxygenases in human and mouse skin and cultured human keratinocytes: association of COX-2 expression with human keratinocyte differentiation. Exp Cell Res 1996; 224: 79-87.

18. Qin H, Wang J, Liang Y, Taniguchi Y, Tanigaki K, Han H. RING1 inhibits transactivation of RBP-J by Notch through interaction with LIM protein KyoT2. Nucleic Acids Res 2004; 32 1492-1501.

19. Grossi M, Hiou-Feige A, Tommasi Di Vignano A, Calautti E, Ostano P et al. Negative control of keratinocyte differentiation by Rho/CRIK signaling coupled with up-regulation of KyoT1/2 (FHL1) expression. Proc Natl Acad Sci USA 2005; 102: 11313-11318.

20. Nguyen BC, Lefort K, Mandinova A, Antonini D, Devgan V, Della Gatta G et al. Crossregulation between Notch and p63 in keratinocyte commitment to differentiation. Genes Dev 2006; 20: 1028-1042.

21. Pearton DJ, Nirunsuksiri W, Rehemtulla A, Lewis SP, Presland RB, Dale BA. Proprotein convertase expression and localization in epidermis: evidence for multiple roles and substrates. Exp Dermatol 2001; 10: 193-203.

22. Emami N, Diamandis EP. Human kallikrein-related peptidase 14 (KLK14) is a new activato component of the KLK proteolytic cascade. Possible function in seminal plasma and skin. $J$ Biol Chem 2008; 283: 3031-3041.

23. Cassidy AJ, van Steensel MA, Steijlen PM, van Geel M, van der Velden J, Morley SM et al. A homozygous missense mutation in TGM5 abolishes epidermal transglutaminase 5 activity and causes acral peeling skin syndrome. Am J Hum Genet 2005; 77 : 909-917.

24. Hohenester E. Structural insight into Slit-Robo signalling. Biochem Soc Trans 2008; 36 251-256.

25. Zhang Y, Andl T, Yang SH, Teta M, Liu F, Seykora JT et al. Activation of beta-catenin signaling programs embryonic epidermis to hair follicle fate. Development 2008; 135 : 2161-2172.

26. Yang L, Yamasaki K, Shirakata Y, Dai X, Tokumaru S, Yahata Y et al. Bone morphogenetic protein-2 modulates Wnt and frizzled expression and enhances the canonical pathway of Wnt signaling in normal keratinocytes. J Dermatol Sci 2006; 42: 111-119.

27. Kazanskaya O, Glinka A, del Barco Barrantes I, Stannek P, Niehrs C, Wu W. R-Spondin2 is a secreted activator of Wnt/beta-catenin signaling and is required for Xenopus myogenesis. Dev Cell 2004; 7: 525-534.

28. Neufang G, Furstenberger G, Heidt M, Marks F, Muller-Decker K. Abnormal differentiation of epidermis in transgenic mice constitutively expressing cyclooxygenase-2 in skin. Proc Natl Acad Sci USA 2001; 98: 7629-7634.
29. Grose R, Harris BS, Cooper L, Topilko P, Martin P. Immediate early genes krox-24 and krox-20 are rapidly up-regulated after wounding in the embryonic and adult mouse. Dev Dyn 2002; 223: 371-378.

30. Longworth MS, Wilson R, Laimins LA. HPV31 E7 facilitates replication by activating E2F2 transcription through its interaction with HDACs. EMBO J 2005; 24: 1821-1830.

31. Rotzer D, Krampert M, Sulyok S, Braun S, Stark HJ, Boukamp P et al. Id proteins: nove targets of activin action, which regulate epidermal homeostasis. Oncogene 2006; 25: 2070-2081.

32. Stelnicki EJ, Kömüves LG, Kwong AO, Holmes D, Klein P, Rozenfeld S et al. HOX homeobox genes exhibit spatial and temporal changes in expression during human skin development. J Invest Dermatol 1998; 110: 110-115.

33. Ford R, Wang G, Jannati P, Adler D, Racanelli P, Higgins PJ et al. Modulation of SPARC expression during butyrate-induced terminal differentiation of cultured human keratinocytes: regulation via a TGF-beta-dependent pathway. Exp Cell Res 1993; 206: 261-275

34. Szepietowski JC, Reich A, McKenzie RC. The multifunctional role of leukaemia inhibitory factor in cutaneous biology. Acta Dermatovenerol Alp Panonica Adriat 2004; 13: 125-129.

35. Choi SY, Shin HC, Kim SY, Park YW. Role of TMPRSS4 during cancer progression. Drug News Perspect 2008; 21: 417-423.

36. Zhang $\mathrm{H}$, Zhang Y, Duan HO, Kirley SD, Lin SX, McDougal WS et al. TIP30 is associated with progression and metastasis of prostate cancer. Int J Cancer 2008; 123: 810-816.

37. Jenkins TD, Opitz OG, Okano J, Rustgi AK. Transactivation of the human keratin 4 and Epstein-Barr virus ED-L2 promoters by gut-enriched Krüppel-like factor. J Biol Chem 1998; 273: 10747-10754

38. Tanioka M, Miyagawa-Hayashino A, Manabe T, Toichi E, Miyachi Y, Takahashi K Circumscribed palmo-plantar hypokeratosis: a disease with two subtypes. J Invest Dermatol 2009; 129: 1045-1047.

39. Martinsson H, Yhr M, Enerbäck C. Expression patterns of S100A7 (psoriasin) and S100A9 (calgranulin-B) in keratinocyte differentiation. Exp Dermatol 2005; 14: 161-168.

40. Eckert RL, Sturniolo MT, Jans R, Kraft CA, Jiang H, Rorke EA. TIG3: a regulator of type I transglutaminase activity in epidermis. Amino Acids 2009; 36: 739-746.

41. Yu AS, Enck AH, Lencer WI, Schneeberger EE. Claudin-8 expression in Madin-Darby canine kidney cells augments the paracellular barrier to cation permeation. J Biol Chem 2003; 278: 17350-17359.

42. Bommer GT, Jäger C, Dürr EM, Baehs S, Eichhorst ST, Brabletz T et al. DRO1, a gene downregulated by oncogenes, mediates growth inhibition in colon and pancreatic cancer cells. J Biol Chem 2005; 280: 7962-7975.

43. Budanov AV, Karin M. p53 target genes sestrin1 and sestrin2 connect genotoxic stress and mTOR signaling. Cell 2008; 134: 451-460.

44. Chau BN, Diaz RL, Saunders MA, Cheng C, Chang AN, Warrener P et al. Identification of SULF2 as a novel transcriptional target of p53 by use of integrated genomic analyses. Cancer Res 2009; 69: 1368-1374.

45. Vogler R, Sauer B, Kim DS, Schäfer-Korting M, Kleuser B. Sphingosine-1-phosphate and its potentially paradoxical effects on critical parameters of cutaneous wound healing. $J$ Invest Dermatol 2003; 120: 693-700.

46. Gartel AL, Goufman E, Tevosian SG, Shih H, Yee AS, Tyner AL. Activation and repression of p21(WAF1/CIP1) transcription by RB binding proteins. Oncogene 1998; 17: 3463-3469.

47. Maurelli R, Zambruno G, Guerra L, Abbruzzese C, Dimri G, Gellini M et al. Inactivation of p16INK4a (inhibitor of cyclin-dependent kinase 4A) immortalizes primary human keratinocytes by maintaining cells in the stem cell compartment. FASEB $J$ 2006; 20: $1516-1518$

48. Su X, Cho MS, Gi YJ, Ayanga BA, Sherr CJ, Flores ER. Rescue of key features of the p63null epithelial phenotype by inactivation of Ink4a and Arf. EMBOJ 2009; 28: 1904-1915.

49. Ogata A, Shimizu T, Abe R, Shimizu H, Sakai M. Expression of c-maf and mafB genes in the skin during rat embryonic development. Acta Histochem 2004; 106: 65-67.

Supplementary Information accompanies the paper on Cell Death and Differentiation website (http://www.nature.com/cdd) 